\title{
RADIATIVE MECHANISMS IN GRB PROMPT EMISSION
}

\author{
A. Pe'er ${ }^{1}$
}

\begin{abstract}
Motivated by the Fermi gamma-ray space telescope results, in recent years immense efforts were given to understanding the mechanism that leads to the prompt emission observed. The failure of the optically thin emission models (synchrotron and synchrotron self Compton) increased interest in alternative models. Optically thick models, while having several advantages, also face difficulty in capturing several key observables. Theoretical efforts are focused in two main directions: (1) mechanisms that act to broaden the Planck spectrum; and (2) combining the optically thin and optically thick models to a hybrid model that could explain the key observables.
\end{abstract}

\section{Setting the stage: Understanding what we see}

In the commonly accepted gamma-ray bursts (GRB) "fireball" model (Goodman 1986; Paczynski 1986; Rees \& Meszaros 1992, 1994; Shemi \& Piran 1990), the prompt emission is believed to arise from a prompt dissipation of a substantial fraction of the bulk kinetic energy of a relativistic outflow, originating from a central compact object. This model is found to be in good qualitative agreement with all observations to date; moreover, a great success of this model is the prediction of the afterglow emission, resulting from interaction of the propagating relativistic blast wave with the ambient interstellar matter (ISM) (Mészáros \& Rees 1997; Sari et al. 1998).

In spite of these successes, this model is far from being complete. Many necessary details are missing: for example, the mechanism responsible for particle acceleration to high energies, required to explain the observed high-energy nonthermal emission is not explained. Similarly, the nature of the radiative processes that produce the observed signal are not specified. In addition, the dynamical part is not fully understood. While it was long thought that the conversion of explosion (gravitational) energy to kinetic energy (namely, acceleration to relativistic velocities) is mediated by photons (Paczynski 1986, 1990; Rees \& Meszaros 1992),

${ }^{1}$ Physics Department, University College Cork, Cork, Ireland 
in recent years there are accumulating evidence that magnetic field may play an important role in this process (Zhang \& Pe'er 2009), resulting in a modified dynamics (Drenkhahn 2002; Drenkhahn \& Spruit 2002). Moreover, nothing in the model predicts the radii in which energy is dissipated and radiation is produced.

The prompt GRB spectra is well modeled by a smoothly broken power law, known as the "Band" function (Band et al. 1993; Goldstein et al. 2012; Kaneko et al. 2006; Nava et al. 2011; Preece et al. 2000, 1998b). In spite of its great success in providing good fits to the observed data, this model has a crucial drawback: being mathematical in nature, by itself it does not provide any clue about the origin of the observed emission.

It was long thought that the observed radiation originates from synchrotron emission in the optically thin regime (Cohen et al. 1997; Mészáros et al. 1993, 1994; Tavani 1996). This idea was motivated by the fact that the observed radiation is non-thermal. Shock waves which are believed to exist in the plasma can accelerate particles to high energies via Fermi mechanism as well as generate strong magnetic fields, thereby providing the necessary ingredients for synchrotron emission (Blandford \& Eichler 1987). These processes were recently realized in particle-incell (PIC) simulations (Haugbølle 2011; Sironi \& Spitkovsky 2009, 2011; Spitkovsky 2008).

Although GRB spectra significantly vary from burst to burst and frequently within a single burst, there are several key observations which appear general. The synchrotron theory can therefore be confronted with these key results. These include:

1. Observed peak energy $E_{\text {peak }}^{o b} \sim 300 \mathrm{keV}$. While the synchrotron theory does not naturally provide this value, it is achievable under the assumption that both the electrons and magnetic field energies are close to equipartition with the post-shock thermal energy. For example, if the magnetic field is $B \approx$ $10^{5} \mathrm{G}$, the characteristic electron's Lorentz factor is $\gamma_{e l} \sim 200$ and bulk Lorentz factor $\Gamma \sim 10^{2.5}$, similar values are obtained.

2. Narrow distribution of the peak energy: although the observed luminosity varies by several orders of magnitude, in most GRBs the observed peak energy is between $0.1-1 \mathrm{MeV}$. In the context of the synchrotron model, the observed peak energy is a function of $B, \gamma_{e l}$ and $\Gamma$. There is no natural reason to assume that the values of these free model parameters coincide in such a way as to produce the narrow clustering of $E_{\text {peak }}^{o b}$ observed.

3. The correlation seen between the peak energy and total energy ( $E_{\text {peak }}-E_{\text {iso }}$ relation) (Amati et al. 2002; Ghirlanda et al. 2004; Golenetskii et al. 1983; Yonetoku et al. 2004): in the framework of the synchrotron model, it is possible to obtain the observed correlation only if additional assumptions are made, e.g., about the dissipation radius.

4. A "universal" low energy spectral slope, $\alpha \approx-1$ (Goldstein et al. 2012; Kaneko et al. 2006; Nava et al. 2011): in the "Band" model fits, a narrow 
clustering of the low energy spectral slope $\left(d N / d E \propto E^{\alpha}\right)$ around $\alpha \approx-1$ is observed. The observed low energy hard spectral slope is in contradiction to the prediction of the synchrotron model theory. This is known as "synchrotron (model) line of death" (Ghirlanda et al. 2003; Preece et al. 2002, 1998a).

The failure of the synchrotron model has motivated the study of alternatives. A notable alternative is emission from the optically thick regions. While many of the details of the "fireball" model are uncertain, the existence of an optically thick region in the inner parts of the outflow is a robust prediction. Thus, photospheric emission is a natural outcome of the model, and, indeed was considered from the very early days (Goodman 1986; Paczynski 1986). However, as the observed spectrum does not resemble a Planck spectrum, this idea was abandoned for a long time.

\section{Broadening mechanisms of Planck spectrum: Sub photospheric energy dissipation}

The observed low energy spectrum is steeper than synchrotron model predictions, but is not as steep as to resemble a "Planck" spectrum. However, while there is no physical mechanism that can steepen the synchrotron spectra, one can think of several mechanisms that can broaden the Planck spectrum to produce the observed spectral slope.

Broadly speaking, there can be three ways in which the observed spectra can be achieved. First, the spectrum may contain two separate components: a "Planck" and optically thin synchrotron observed simultaneously. The observed spectrum is a combination of these two components. Following early analysis by Ryde (2004, 2005) and Ryde \& Pe'er (2009), recently, with improved Fermi capabilities that enable time-resolved analysis, these components are ubiquitously observed (Axelsson et al. 2012; Guiriec et al. 2011, 2012; Larsson et al. 2011; Ryde et al. 2010; Starling et al. 2012; Zhang et al. 2011). The separation enables the study of the physical properties of both components (Pe'er et al. 2007, 2012; Zhao et al. 2011), and provides a natural explanation to the delay of the high energy emission seen (Abdo et al. 2009; Ackermann et al. 2010).

Second, sub-photospheric energy dissipation naturally leads to modification of the Planck spectrum (Beloborodov 2010; Giannios 2006, 2008, 2012; Ioka et al. 2007; Lazzati \& Begelman 2010; Lazzati et al. 2009; Pe'er et al. 2005, 2006; Vurm et al. 2011). The basic idea is that kinetic energy dissipation, whether originating from internal shocks, magnetic reconnection or any other process, takes place at radii not much below the photospheric radius. By definition of the photospheric radius $r_{p h}$, the optical depth for scattering of a photon from $r_{p h}$ to the observer (located at infinity) is equal to unity. The plasma contains many more photons than electrons: this can be seen by the fact that the average energy per photon (in the comoving frame) is much smaller than $m_{e} c^{2}$. Thus, while at $r_{p h}$ the optical depth for photon scattering is unity, the optical depth for electron scattering is 
much larger than unity. As a result, at $r_{p h}$, every electron undergoes many inverse Compton (IC) scatterings with the lower energy photons before decoupling. Each electron therefore loses its energy rapidly, on a time scale much shorter than the dynamical (expansion) time scale (see Pe'er et al. 2005 for details).

Assuming that the heating mechanism (of an unspecified nature) is continuously heating the electrons, or alternatively accelerating new electrons to high energies, the result is that the electron's distribution is in a quasi steady state, with temperature determined by balance between the external heating and the rapid IC cooling. This temperature is inevitably higher than the photon temperature, hence the plasma is characterized by two temperatures: $T_{e l}>T_{p h}$.

If the dissipation, hence the electron heating occurs below, or even slightly above the photosphere, then the thermal photons IC scatter with the hotter electrons, producing a non-thermal spectrum. The emerging spectrum above the original thermal peak depends mainly on two free model parameters: (1) the optical depth $\tau$ in which the dissipation takes place: this determines the number of scattering for a single photon. On the one extreme, $\tau \rightarrow \infty$ (or $r_{d i s} \ll r_{p h}$ ), the plasma have enough time to thermalize, and the energy given to the electrons is evenly distributed, resulting in a Planck spectrum. On the other extreme, $\tau \ll 1$, only very few photons are being up scattered, producing a high energy tail. In the intermediate regime, $\tau \approx$ few - few tens, the spectrum significantly deviates from Planck. (2) The second free parameter is the ratio of the energy density in the electron and thermal photon components. If the dissipation considerably heats the electrons, deviation from a Planck spectrum is more pronounced.

Multiple IC scattering thus modifies the spectrum above the thermal peak. At lower frequencies, the spectrum is dominated by synchrotron emission from the energetic electrons. As these electrons are in a quasi steady state, the emerging spectrum does not expect to have a power law shape, as the electrons distribution cannot be described by a power law. Thus, overall, the expected spectra is expected to significantly deviate from the original Planck spectra, with significant synchrotron contribution at low energies, and high energy spectra dominated by multiple IC scatterings. Example of possible spectra under different conditions appear in Figure 1, taken from Pe'er et al. (2006). Recently, evidence for sub-photospheric energy dissipation was observed in analyzing the data of GRB 090902B (Ryde et al. 2011).

\section{Theory of photospheric emission from collimated outflow}

Even in the absence of sub-photospheric energy dissipation, the expected spectrum originating from the photosphere deviates from a pure "Planck" spectrum. This is due to the non-trivial shape of the photosphere. Consider first a spherical explosion: the mean free path of photons emitted from high angle to the line of sight, $\theta>0$ and propagate towards the observer is larger than the mean free path of photons propagating at $\theta=0$. This results in a strong angular dependence of the photospheric radius, $r_{p h} \propto \Gamma^{-2}+\theta^{2} / 3$ (Pe'er 2008), where $\Gamma$ is the bulk Lorentz factor. 


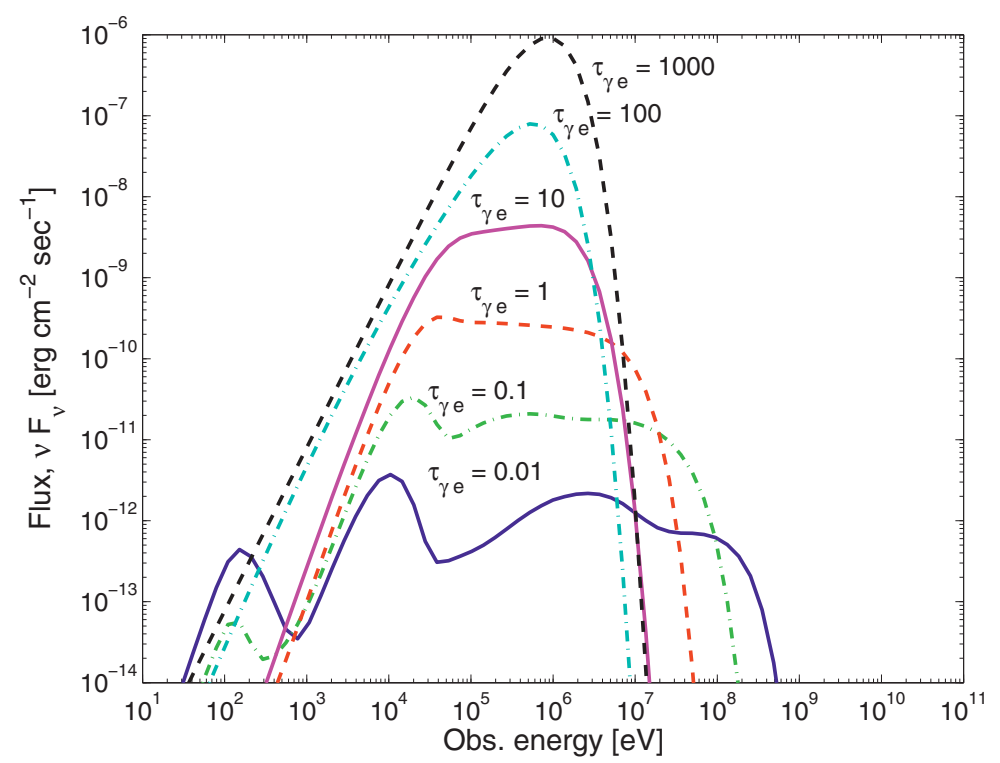

Fig. 1. Examples of time averaged spectra obtained for different values of the optical depth for photon scattering $\left(\tau=\tau_{\gamma e}\right)$ at the dissipation radius, under the assumption that thermal component exists (from Pe'er et al. 2006). While for very high optical depth a "Planck" spectrum is obtained, for intermediate optical depth, multiple IC scattering results in nearly flat spectra above the thermal peak, while synchrotron emission modifies the spectrum at lower energies.

Moreover, by definition, the photospheric radius is the radius in which the optical depth for scattering $\tau=1$. However, the last scattering process is not limited to this surface: photons have a finite probability of being scattered at any position in space in which scatterers (electrons) exist. An observer therefore sees simultaneously photons who's last scattering location took place at a range of radii and angles to the line of sight; this leads to the concept of a "fuzzy photosphere". As photons adiabatically cool below the photosphere, each of the observed photons has its own (comoving) energy, and has a unique Doppler boost. Thus, the observed spectrum differs than Planck spectrum, and is observed as a "gray body" spectrum (Beloborodov 2011; Pe'er 2008). If one considers a $\delta$-function of emission at $t=0$ (alternatively, if the inner engine is abruptly stopped), then at late times emission is dominated by photons emitted at high angles (off-axis). In this scenario, a very flat spectrum is obtained at late times, significantly different than a "Planck" (Pe'er \& Ryde 2011).

While the original theory was developed for spherical outflows, in any realistic scenario the explosion is collimated. In the collapsar model, for example, as the jet drills its way through the collapsing stellar envelope it pushes material towards 
the side, forming a hot cocoon. This material collimates the jet (Mizuta et al. 2011; Morsony et al. 2007; Zhang et al. 2003). Thus, when calculating emission from the photosphere one needs to consider the jet velocity profile, $\Gamma=\Gamma(\theta)$. Such a model therefore has 4 free parameters (as opposed to a single parameter, $\Gamma$ in the spherical case): the maximum bulk Lorentz factor $\Gamma_{0}$ at the jet axis, the characteristic jet opening angle $\theta_{j}$, viewing angle $\theta_{v}$ and a parameter $p$ which determines the shape of the velocity profile decay $\left(\Gamma(\theta) \propto \theta^{-p}\right)$.

Such a scenario was recently studied by Lundman et al. (2013). It leads to a few unexpected results. First, extended emission from higher angles is very pronounced. This can be understood as a phase space effect: the average scattering angle is $\approx \Gamma^{-1}$, and $\Gamma$ varies with angle. Thus, more photons that originates from high angles (with lower $\Gamma$ ) are observed, compared to the spherical case. The obtained spectrum for narrow jet $\left(\theta_{j} \Gamma_{0} \lesssim\right.$ few $)$ below the thermal peak is flat $\left(d N / d E \propto E^{-1}\right)$, independent of viewing angle, and only weakly dependent on the Lorentz factor gradient $(p)$. A similar result is obtained for wider jets, observed at $\theta_{v} \approx \theta_{j}$, which is the most likely scenario. The spectral slope calculated in this model is similar to the average low energy spectral slope observed. For wider jets $\left(\theta_{j} \Gamma_{0} \gtrsim\right.$ few $)$, a multicolor black body is obtained. Second, the high energy spectral slope is modified by a similar mechanism: as the average scattering angle is $\approx \Gamma^{-1}$, photons are more likely to diffuse from region of low $\Gamma$ to region of high $\Gamma$, where they are further boosted. This leads to a power law spectral slope at high energies, who's exact shape depends on the assumed jet profile. An example of the obtained spectra appears in Figure 2.

\section{Summary}

In spite of two decades of extensive research, the origin of GRB prompt emission remains elusive. A renewed interest in understanding this phenomena occurred with the superb data quality enabled by the Fermi satellite. Following the failure of the synchrotron model, significant efforts were given to understanding mechanisms that can act to broaden the Planck spectrum to fit into the observed "Band" spectrum.

Three ideas were suggested in recent years: (1) A combined optically thick and optically thin emission seen simultaneously; (2) sub-photospheric energy dissipation; and (3) geometrical broadening. While each of these ideas have its own success, as of today, non of these provide a full explanation to the observed spectrum. The success and weaknesses of any of these ideas are summarized in Table 1 below. In the table, $(V)$ represents success, $(X)$ represents failure, and $(-)$ implies that currently the theory does not contradict the observation, but does not provide predictions either, or that additional assumptions are required.

Thus, as of today, no single model can fully explain all key observations, implying plenty of room for new ideas.

I would like to thank my collaborator and friend Felix Ryde for countless number of useful discussions. 


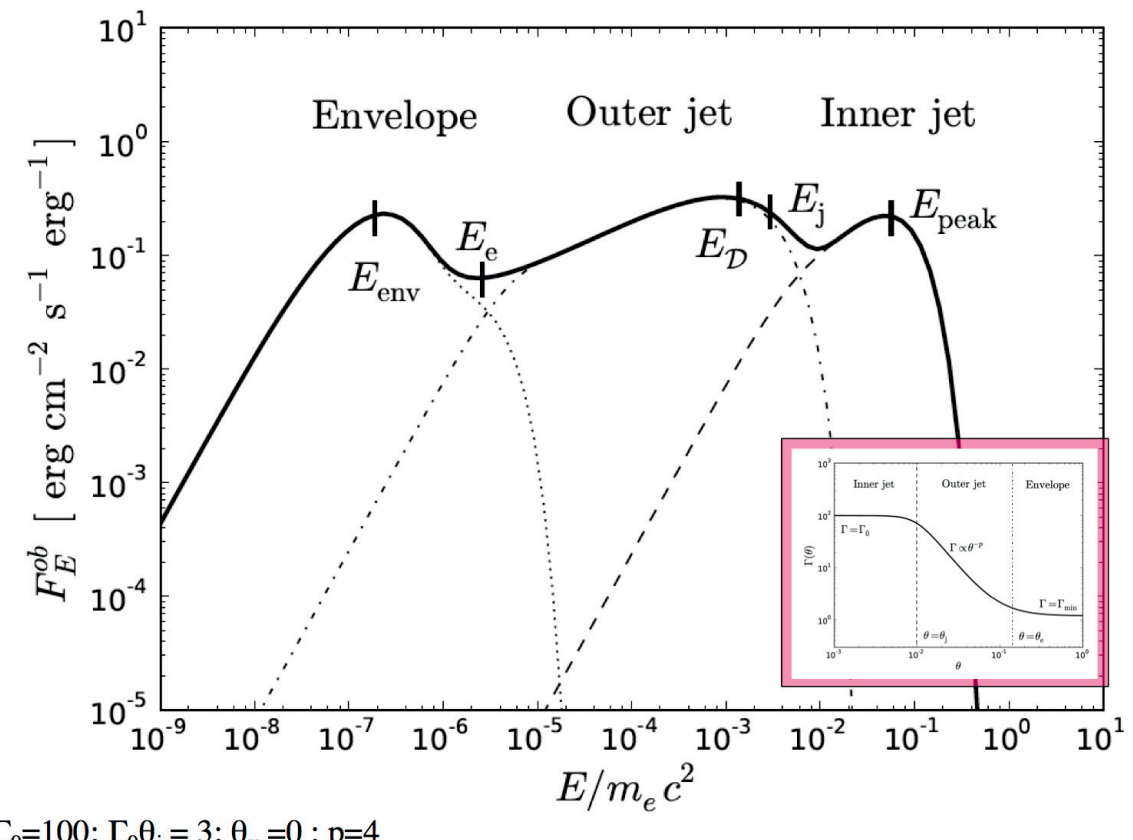

Fig. 2. Example of observed spectrum from a relativistic, optically thick outflow (taken from Lundman et al. 2013). A jet profile $\left.\Gamma(\theta) \propto \Gamma_{0} /\left(\theta / \theta_{j}\right)^{2 p}+1\right)^{1 / 2}$ was considered (inner onset). Separate contributions from the inner jet $\left(\Gamma \approx\right.$ const), outer jet $\left(\Gamma \propto \theta^{-p}\right)$ and envelope $(\Gamma \gtrsim 1)$ are marked. The combined effect is a very flat spectra, extended over many orders of magnitude. This result is found to be robust, very weakly dependent on the values of the free model parameters.

Table 1. Confronting current theoretical models with key observations.

\begin{tabular}{|c|ccccc|}
\hline \hline Key observation & \multicolumn{4}{|c}{ Optically thin Pure Planck Sub phot. energy } & Geometrical \\
& synchrotron & + synch. & dissipation & broadening \\
\hline$E_{\text {peak }}^{o b} \approx 300 \mathrm{keV}$ & $\mathrm{V}$ & $\mathrm{V}$ & $\mathrm{V}$ & $-\mathrm{V}$ \\
Narrow $E_{\text {peak }}^{\text {ob }}$ & - & - & $\mathrm{V}$ & - \\
distribution & & $\mathrm{X}-$ & $\mathrm{X}-$ & $\mathrm{X}-$ \\
$\begin{array}{c}E_{\text {peak }}-E_{\text {iso }} \\
\text { correlation }\end{array}$ & - & $\mathrm{X}$ & & $\mathrm{V}$ \\
$\begin{array}{c}\text { Low energy spectral } \\
\text { index }<\alpha>\approx-1\end{array}$ & $\mathrm{X}$ & $\mathrm{X}$ & & \\
\hline
\end{tabular}

\section{References}

Abdo, A.A., Ackermann, M., Ajello, M., et al., 2009, ApJ, 706, L138

Ackermann, M., Asano, K., Atwood, et al., 2010, ApJ, 716, 1178 
Amati, L., Frontera, F., Tavani, M., et al., 2002, A\&A, 390, 81

Axelsson, M., Baldini, L., Barbiellini, G., et al., 2012, ApJ, 757, L31

Band, D., Matteson, J., Ford, L., et al., 1993, ApJ, 413, 281

Beloborodov, A.M., 2010, MNRAS, 407, 1033

Beloborodov, A.M., 2011, ApJ, 737, 68

Blandford, R., \& Eichler, D., 1987, Phys. Rep., 154, 1

Cohen, E., Katz, J.I., Piran, et al., 1997, ApJ, 488, 330

Drenkhahn, G., 2002, A\&A, 387, 714

Drenkhahn, G., \& Spruit, H.C., 2002, A\&A, 391, 1141

Ghirlanda, G., Celotti, A., \& Ghisellini, G., 2003, A\&A, 406, 879

Ghirlanda, G., Ghisellini, G., \& Lazzati, D., 2004, ApJ, 616, 331

Giannios, D., 2006, A\&A, 457, 763

Giannios, D., 2008, A\&A, 480, 305

Giannios, D., 2012, MNRAS, 422, 3092

Goldstein, A., Burgess, J.M., Preece, R.D., et al., 2012, ApJS, 199, 19

Golenetskii, S.V., Mazets, E.P., Aptekar, R.L., \& Ilinskii, V.N., 1983, Nature, 306, 451

Goodman, J., 1986, ApJ, 308, L47

Guiriec, S., Connaughton, V., Briggs, M.S., et al., 2011, ApJ, 727, L33

Guiriec, S., Daigne, F., Hascoët, R., et al., 2012 [ArXiv e-prints 1210.7252]

Haugbølle, T., 2011, ApJ, 739, L42

Ioka, K., Murase, K., Toma, K., Nagataki, S., \& Nakamura, T., 2007, ApJ, 670, L77

Kaneko, Y., Preece, R.D., Briggs, M.S., et al., 2006, ApJS, 166, 298

Larsson, J., Ryde, F., Lundman, C., et al., 2011, MNRAS, 414, 2642

Lazzati, D., \& Begelman, M.C., 2010, ApJ, 725, 1137

Lazzati, D., Morsony, B.J., \& Begelman, M.C., 2009, ApJ, 700, L47

Lundman, C., Pe'er, A., \& Ryde, F., 2013, MNRAS, 428, 2430

Meszaros, P., Laguna, P., \& Rees, M.J., 1993, ApJ, 415, 181

Meszaros, P., \& Rees, M.J., 1997, ApJ, 476, 232

Mészáros, P., Rees, M.J., \& Papathanassiou, H., 1994, ApJ, 432, 181

Mizuta, A., Nagataki, S., \& Aoi, J., 2011, ApJ, 732, 26

Morsony, B.J., Lazzati, D., \& Begelman, M.C., 2007, ApJ, 665, 569

Nava, L., Ghirlanda, G., Ghisellini, G., \& Celotti, A., 2011, A\&A, 530, A21

Paczynski, B., 1986, ApJ, 308, L43

Paczynski, B., 1990, ApJ, 363, 218

Pe'er, A., 2008, ApJ, 682, 463

Pe'er, A., Mészáros, P., \& Rees, M.J., 2005, ApJ, 635, 476

Pe'er, A., Mészáros, P., \& Rees, M.J., 2006, ApJ, 642, 995

Pe'er, A., \& Ryde, F., 2011, ApJ, 732, 49

Pe'er, A., Ryde, F., Wijers, R.A.M.J., et al., 2007, ApJ, 664, L1

Pe'er, A., Zhang, B.-B., Ryde, F., et al., 2012, MNRAS, 420, 468

Preece, R.D., Briggs, M.S., Giblin, T.W., et al., 2002, ApJ, 581, 1248

Preece, R.D., Briggs, M.S., Mallozzi, R.S., et al., 1998a, ApJ, 506, L23 
Preece, R.D., Briggs, M., Mallozzi, R.S., et al., 2000, ApJS, 126, 19

Preece, R.D., Pendleton, G.N., Briggs, M.S., et al., 1998b, ApJ, 496, 849

Rees, M.J., \& Meszaros, P., 1992, MNRAS, 258, 41P

Rees, M.J., \& Meszaros, P., 1994, ApJ, 430, L93

Ryde, F., 2004, ApJ, 614, 827

Ryde, F., 2005, ApJ, 625, L95

Ryde, F., Axelsson, M., Zhang, B., et al., 2010, ApJ, 709, L172

Ryde, F., \& Pe'er, A., 2009, ApJ, 702, 1211

Ryde, F., Pe'er, A., Nymark, T., et al., 2011, MNRAS, 415, 3693

Sari, R., Piran, T., \& Narayan, R., 1998, ApJ, 497, L17

Shemi, A., \& Piran, T., 1990, ApJ, 365, L55

Sironi, L., \& Spitkovsky, A., 2009, ApJ, 707, L92

Sironi, L., \& Spitkovsky, A., 2011, ApJ, 726, 75

Spitkovsky, A., 2008, ApJ, 682, L5

Starling, R.L.C., Page, K.L., Pe'er, A., Beardmore, A.P., \& Osborne, J.P., 2012, MNRAS, 427,2950

Tavani, M., 1996, ApJ, 466, 768

Vurm, I., Beloborodov, A.M., \& Poutanen, J., 2011, ApJ, 738, 77

Yonetoku, D., Murakami, T., Nakamura, T., et al., 2004, ApJ, 609, 935

Zhang, B., \& Pe'er, A., 2009, ApJ, 700, L65

Zhang, B.-B., Zhang, B., Liang, E.-W., et al., 2011, ApJ, 730, 141

Zhang, W., Woosley, S.E., \& MacFadyen, A.I., 2003, ApJ, 586, 356

Zhao, X.-H., Li, Z., \& Bai, J.-M., 2011, ApJ, 726, 89 
